\begin{tabular}{|c|l|}
\hline Title & $\begin{array}{l}\text { Linear viscoel astic analysis using frequency-domain algorithm on oscillating circular shear flows for bubble } \\
\text { suspensions }\end{array}$ \\
\hline Author(s) & Tasaka, Y uji; Y oshida, Taiki; Rapberger, Richard; Murai, Y uichi \\
\hline Citation & $\begin{array}{l}\text { Rheologica A cta, 57/3), 229-240 } \\
\text { https://doi.org/10.1007/300397-018-1074-z }\end{array}$ \\
\hline Issue Date & 2018-03 \\
\hline Doc URL & http://hdl.handle.net/2115/2705 \\
\hline Rights & "The final publication is available at link.springer.com". \\
\hline Type & article (author version) \\
\hline File Information & FFT \\
\hline
\end{tabular}

Instructions for use 


\title{
Linear viscoelastic analysis using frequency-domain algorithm on oscillating circular shear flows for bubble suspensions
}

\author{
Yuji Tasaka - Taiki Yoshida • Richard Rapberger · Yuichi Murai
}

Received: date / Revised version: date

\begin{abstract}
To achieve a stable evaluation of the linear viscoelasticity of bubble suspensions, which have difficulties for conventional rheometers from spatial distributions of rheological properties with bubble deformations, we proposed a novel rheometry based on spatiotemporal velocity data obtained by ultrasonic velocity profiling (UVP). A frequency-domain algorithm was adopted to overcome a critical influence of measurement noise on the rheological assessment, which is inferred from error propagation characteristics through the equations of motion in discretized form. Applicability and advantage of the present rheometry with the frequency-domain algorithm were verified by two kinds of fluids; high viscous oil as a Newtonian fluid, and polyacrylamide aqueous solution as a shear thinning, viscoelastic fluid. The rheometry was finally adopted for bubble suspensions subject to high oscillatory shear, and it could validly extract elasticity-originated momentum transfer as a function of space.
\end{abstract}

Keywords Rheometry · Ultrasonic velocimetry · Linear viscoelasticity · Bubble suspensions

\section{Introduction}

Regarding aspects such as quality control of products, safety issues, and more efficient processing in any indus-

Y. Tasaka, T. Yoshida and Y. Murai

Laboratory for Flow Control, Faculty of Engineering,

Hokkaido University, Sapporo, Japan

Tel.: +81-11-7066371

Fax: +81-11-7066373

E-mail: tasaka@eng.hokudai.ac.jp

R. Rapberger

Institute for Design and Control of Mechatronic Systems, Johannes Kepler University in Linz, Linz, Austria trial field, details of rheological properties of fluid media are required. The developments of highly precise torque meters has made it possible to provide good estimations of such details. Along with rheological models that are constitutive equations describing the stress response in materials, rotational rheometry has provided details of properties using only a small number of characteristic constants. Nevertheless, rotational rheometry assumes simple Couette flows produced in a narrow gap between stator and rotor connected to torque meters. However, dispersed multiphase media, which are not seen as continuum even at macroscopic scale, are difficult to measure. Non-ideal conditions run counter to the assumption of Couette flows required to solve the "Couette inverse problem" to obtain the original rheological properties (e.g. Yeow et al. (2000); Ancey (2005); Heirman et al. (2008)). Furthermore, in measurements of multiphase media, additional complexities arise for example from the presence of interfaces in gap size (e.g., Doi and Ohta (1991); Stickel and Powell (2005)).

A focus of our studies on the rheology of multiphase media is bubble suspensions for understanding the mechanisms underpinning drag reduction from injected bubbles (e.g., Ceccio (2010); Murai (2014)). Bubbles accumulated in flow elements, strong shear layers and vortices, create locally different rheological properties in conditions of unsteady shear flow in turbulence. Beginning with the appearance of the classical theory on dilute spherical suspensions (assuming that surface tension is strong enough to sustain the spherical shape) in Einstein (1906) and Taylor (1932), the study of suspension rheology has a century-long history. By considering capillary number, the theory was extended to deformable bubbles in simple steady shear flows (Frankel and Acrivos (1970)), and its applicability was validated by experiments (Rust and Manga (2002)). Choi and 
Schowalter (1975) provided a more sophisticated equation that considered higher orders in the volume fraction to extend the theory to larger volume fractions. Despite the progress made over the century, there remain aspects to be explained about the elastic response attributed to surface tension effect in unsteady shear flows (Llewellin et al. (2002); Llewellin and Manga (2005); Tasaka et al. (2015)).

For a rheological evaluation of multiphase media, there are two issues to be solved: (1) the narrow-gap problem and (2) the inverse Couette problem. The Stokestype rheometry, for instance, the falling sphere rheometry (bubble suspensions; e.g., see Murai and Oiwa (2008)) and commercial turning sphere rheometry (BMS, Anton Paar $\mathrm{GmbH}$ ), can avoid the issues arising from Couette-type rheometry. However, the rheological properties evaluated using these methods correspond to aggregated rheological properties because of the multidimensional, multicomponent flows dealt with. Thus, the rheological properties obtained are difficult to interpret along standard approaches in rheology.

In solving the issues in regard to Couette-type rheometry, a combination of a wide-gap cylinder system and velocimetry has been tried; spatial profiling of velocity measured in a wider gap using different kinds of velocimetry can reveal deviations to ideal Couette flow that arises from complex rheological properties, for example, shear banding (e.g., Dimitriou et al. (2012)). Supplementing the data by torque measurements to give an integrated boundary condition on a rotor in the system has supported evaluation of complex rheological properties. The kinds of velocimetry adopted include particle image velocimetry (PIV), specially named "RheoPIV" (Rodríguez-González et al. (2010); Dimitriou et al. (2012); Pérez-González et al. (2012); Serrano-Aguilera et al. (2016)), magnetic resonance imaging velocimetry (Jarny et al. (2005); Ovarlez et al. (2005)), laser Doppler velocimetry (Quinzani et al. (1995); Rothstein and Gareth (2002)), and ultrasonic imaging velocimetry (Gurung et al. (2016)).

In contrast to these types of velocimetry, ultrasonic Doppler velocimetry or ultrasonic velocity profiling (UVP) (Takeda (2012)) offers ease of handling and access to opaque fluids. Also UVP is being further developed both in hardware and software (Fischer et al. (2008); Meacci et al. (2016); Muramatsu et al. (2015)), and we can expect in the near future greater sophistication in its methodology. Moreover, recent progress in combining it with Doppler optical coherent tomography has overcome several disadvantages of UVP in nearwall measurements of the velocity field (Salmela et al. (2013)). UVP was applied to a circular Couette system (Murai (2012); Derakhshandeh et al. (2012)) and also to pipe flow with measurements of the pressure drop along the pipe (Ouriev and Windhab (2002); Wiklund and Standing (2008)). The latter technique has been termed the in-line UVP-PD method and has been recognized as a semi-standard evaluation tool in food rheology (Rao et al. (2014)) as ultrasonic velocimetry has been applied avidly in the food processing industry.

Because of its spatio-temporal velocity profiling, UVP has also been explored in visualizing rheological behaviors (Shiratori et al. (2013)). Our group has been developing ultrasonic spinning rheometry (USR) that uses such data to evaluate rheological properties modeled by equations of motion of fluid media and has extended its applicability to viscoelastic analyses of multiphase media including bubble suspensions.

This study evaluates the applicability of USR in linear viscoelastic analysis in general and bubble suspensions, in particular. We investigate the influence of measurement noise on spatio-temporal velocity data measured by UVP that is required for a viscoelastic analysis. We propose a novel algorithm for USR applying Fourier transform theory to the velocity data to achieve more stable analysis. The structure of this paper is as follows: USR including theory and fundamental measurement configuration is briefly summarized in $\S 2$. The applicability of the USR and the numerical experiments in investigating the influence of the measurement noise on USR are presented in $\S 3$. The theory of "frequency-domain analysis" is described. Its applicability to viscosity analysis is evaluated in numerical experiments and on actual velocity data obtained from viscous oil treated as a Newtonian fluid. Then linear viscoelasticity analysis using the algorithm on bubble suspension is performed in $\S 4$. Finally, concluding remarks are presented in $\S 5$.

\section{Ultrasonic spinning rheometry (USR)}

\subsection{Measurement configuration}

The USR process involves two main steps, the measurements of the velocity profile of test fluids placed in a cylindrical vessel and the post-processing of the velocity data to evaluate rheological properties. In preparation for explaining the post-processing, brief explanation of the measuring of velocity profiles is given here. The basic configuration of the measurement [Fig. 1(a)] comprises an open-type cylindrical vessel of radius $R$ filled with a test fluid rotating under set conditions. Measurements of the velocity are performed using an ultrasonic transducer (TDX) mounted on the outside of the cylinder. The measurement line for ultrasonic velocity profiling (UVP) (e.g., Takeda (2012)) is set parallel to the 
centerline of the cylinder with a certain displacement of $\Delta y$ to measure the azimuthal component of velocity $u$; UVP measures the on-axis velocity component $u_{\xi}$ along the measurement line $\xi$, and thus the azimuthal velocity component is given as $u=u_{\xi} r / \Delta y$ when the radial velocity component is negligibly small. Test fluids are required to be seeded where there are no ingredients that can scatter ultrasonic waves. Further details of individual measurements are described elsewhere (Tasaka et al. (2015); Yoshida et al. (2017)).

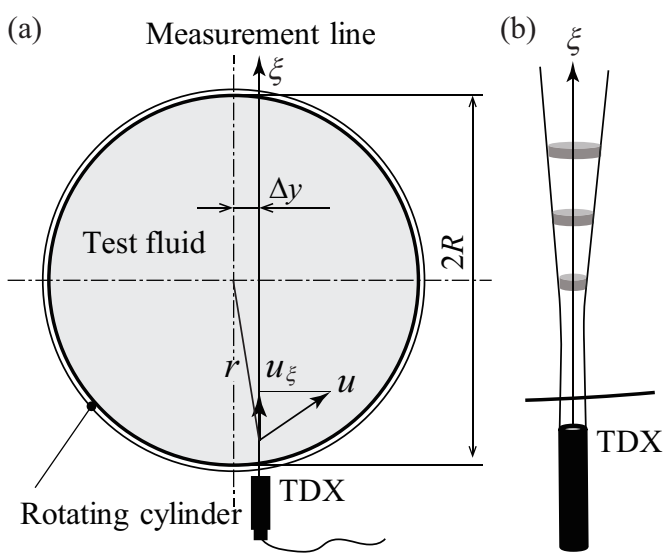

Fig. 1 Schematics of (a) the experimental setup showing the rotating cylinder and the measurement line for ultrasonic velocity profiling (UVP) and (b) the measurement volume of UVP

In measurements by UVP, representative velocities in the disk-shaped volume of the test sample are captured as on-axis velocity components at each radial position along the measurement line [Fig. 1(b)]. The diameter and width of the measurement volume are determined from the size of the Piezo-element in the transducers and the wavelength of the ultrasonic wave in the test fluids; typically these are around $5 \mathrm{~mm}$ and $1 \mathrm{~mm}$, respectively. Velocities $u(r, t)$ calculated from the on-axis velocity component measured in UVP at each volume are processed. They reflects flow behaviors determined by the local characteristics of the test fluids responding to cylinder motions. These characteristics include rheological characteristics, for example, shear-rate-dependent viscosity, viscoelasticity, and non-uniformity of ingredients and local structures. USR extracts from $u(r, t)$ the local characteristics with postprocessing, which shall be summarized below.

\subsection{USR concept and procedure}

USR is concerned with deriving rheological characteristics from spatio-temporal velocity distributions measured by UVP.
Here we summarize the post-processing procedure used in USR. The rheological characteristics of fluids are reflected in the spatio-temporal velocity distributions which are governed by the equation of motions and the constitutive equations (in rheological modeling) describing the relationships between stress $\tau$, strain $\gamma$, and strain rate $\dot{\gamma}$. To simplify the model, we assume just two-dimensional one-directional flows in the azimuthal direction that can be realized in the setup mentioned in the last section. The corresponding equation of motion, Cauchy's equation, is

$\rho \frac{\partial u}{\partial t}=\frac{\partial \tau}{\partial r}+\frac{2 \tau}{r}$

where $\rho$ is the density of the fluid. To determine the rheological properties, Murai (2012) proposed minimizing a cost function expressed by the least-squares approximation,

$F(A, B, C, \cdots)=\left(\rho \frac{\partial u}{\partial t}-\frac{\partial \tau}{\partial r}-\frac{2 \tau}{r}\right)^{2}$,

where the parameters, $A, B, C, \cdots$, denote constants in rheological models representing rheological properties. In the equation above, $u(r, t)$ is given as measurement data in circular shear flows measured by UVP, and $\tau$ is also calculated from $u(r, t)$ through a rheological model adopted.

\section{Influence of measurement error on USR}

\subsection{Viscoelastic analysis of bubble suspensions}

We performed an analysis using the above equations of motion to evaluate viscoelasticity of bubble suspensions having examined their effective Newtonian viscosity in a previous paper (Tasaka et al. (2015)). Experiments measuring velocity profiles were performed with a cylinder of inner diameter $2 R=145 \mathrm{~mm}$ filled with $1000 \mathrm{~mm}^{2} / \mathrm{s}$ silicone oil to a depth of $330 \mathrm{~mm}$. Small bubbles of around 1-mm diameter were dispersed in the fluid layer to a volume fraction of about $2 \%$. The cylinder underwent sinusoidal oscillations of frequency $f_{0}=1 \mathrm{~Hz}$ and a $90^{\circ}$ angular amplitude. The spatiotemporal velocity profiles were captured in ultrasonic velocimetry at a spatial resolution $0.99-\mathrm{mm}$ long the measurement axis and a time resolution of 30-ms.

We recall Cauchy's equation of motion, Eq. 1, and adopt Maxwell's spring-dashpot model in describing viscoelastic fluids,

$\tau+\frac{\mu}{E} \frac{\partial \tau}{\partial t}=\mu\left(\frac{\partial u}{\partial r}-\frac{u}{r}\right)$ 
in establishing the simplest model to describe linear viscoelasticity, which is evaluated by first determining viscosity $\mu$ and elasticity $E$ from measurement data of $u(r, t)$. A suitable set of $\mu$ and $E$ that satisfy these equations is derived as a constraint condition on $\tau$. Mathematically, the calculation is

$$
\begin{array}{r}
\min _{\tau, \mu, E} \int_{r} \int_{t}\left[\tau+\frac{\mu}{E} \frac{\partial \tau}{\partial t}-\mu\left(\frac{\partial u}{\partial r}-\frac{u}{r}\right)\right]^{2} d t d r, \\
\text { s.t. : } \rho \frac{\partial u}{\partial t}-\frac{\partial \tau}{\partial r}-\frac{2 \tau}{r}=0 .
\end{array}
$$

In terms of difference expressions for $u(r, t)$ and $\tau(r, t)$, these become

$$
\begin{aligned}
& \min _{\tau, \mu, E} \sum_{i} \sum_{j}\left[\tau_{i, j}+\frac{\mu}{E} \frac{\tau_{i+1, j}-\tau_{i, j}}{\Delta t}\right.\left.-\mu\left(\frac{u_{i, j+1}-u_{i, j}}{\Delta r}-\frac{u_{i, j}}{r_{j}}\right)\right]^{2}, \\
& \text { s.t. }: \rho \frac{u_{i+1, j}-u_{i, j}}{\Delta t}-\frac{\tau_{i, j+1}-\tau_{i, j}}{\Delta r}-\frac{2 \tau_{i, j}}{r_{j}}=0,
\end{aligned}
$$

where $\Delta t$ and $\Delta r$ correspond to the spatial and radial resolution of the velocity profile measurements. More precisely, the radial positions are calculated from the positions on the measurement line with $\xi$ and $\Delta r$ varying depending on radial positions.

The calculations described above are performed after applying a filter to $u(r, t)$ to suppress the influence of measurement noise. Here we adopt a Savitzky-Golay FIR smoothing filter (Savizky and Golay (1964)) with various filter sizes in time and space to examine the influence of the filter. As radial variations of the viscoelasticity are expected, a narrow radial range of velocity profiles in $r / R=0.86-0.96$ are analyzed over a period of $4 \mathrm{~s}$. The evaluation results for viscosity $\mu$ and elasticity $E$ under different filter sizes are summarized in Table 1 . The phase difference in linear viscoelasticity $\delta$ is defined as the fraction of storage modulus to loss modulus and has following relation with elasticity and viscosity in the Maxwell model,

$\tan \delta=\frac{G^{\prime \prime}}{G^{\prime}}=\frac{E}{2 \pi f_{0} \mu}$.

Phase values of around $90^{\circ}$ mean that a fluid is close to a pure viscous body and smaller values indicate larger elastic contributions to stress in the fluids.

From the various analyses with different filter sizes, designated by the serial number \# in Table 1, the evaluation results of $\mu$ and $E$ are widely scattered. Taking similar values for different analysis, the phase difference $\delta$, however, is stable against the variation in filter size.
This trend may arise from two factors: one that $\mu$ and $E$ appear as a fraction (or product) in the equation to be analyzed, and the other is that the influence of measurement error and noise on $u(r, t)$ including the influence of filtering buries local minimums on the surface of the cost function, Eq. 6, in parameter space. We therefore, evaluated the influence of noise in determining the local minima of the cost function.

\subsection{Numerical evaluation of influence of noise}

For this purpose, we reduce the problem to a Newtonian viscosity analysis. For Newtonian fluids, the equation of motion in Eq. 1 becomes,

$\frac{\partial u}{\partial t}=\nu\left(\frac{\partial^{2} u}{\partial r^{2}}+\frac{1}{r} \frac{\partial u}{\partial r}-\frac{u}{r^{2}}\right)=\nu D_{r} u$,

where $\nu$ is kinematic viscosity and $D_{r}$ is a differential operator with respect to $r$ defined as

$D_{r}=\frac{\partial^{2}}{\partial r^{2}}+\frac{1}{r} \frac{\partial}{\partial r}-\frac{1}{r^{2}}$.

This can be reduced into the cylindrical Bessel differential equation by separation of variables about $t$ and $r$. Then it can be solved by inserting the infinite series

$$
\begin{array}{r}
u(r, t)=\frac{U}{\Phi_{R}^{2}+\Psi_{R}^{2}}\left[\left(\Phi \Phi_{R}+\Psi \Psi_{R}\right) \sin \omega t\right. \\
\left.+\left(\Phi_{R} \Psi-\Phi \Psi_{R}\right) \cos \omega t\right],
\end{array}
$$

where $U$ is angular velocity of the side wall, and

$\Phi(r)=\sum_{m=0}^{\infty} \phi_{m}(r), \quad \Phi_{R}=\sum_{m=0}^{\infty} \phi_{m}(r=R)$,
$\Psi(r)=\sum_{m=0}^{\infty} \psi_{m}(r), \quad \Psi_{R}=\sum_{m=0}^{\infty} \psi_{m}(r=R)$,

and

$$
\begin{gathered}
\phi_{m}(r)=\frac{2^{m}}{m !(m+1) !}\left(\frac{k r}{2}\right)^{2 m+1} f_{m}, \quad k=\sqrt{\frac{\omega}{2 \nu}}, \\
f_{m}= \begin{cases}(-1)^{(m+2) / 2} & : m=\text { even number } \\
(-1)^{(m+1) / 2} & : m=\text { odd number }\end{cases} \\
\psi_{m}(r)=\frac{2^{m}}{m !(m+1) !}\left(\frac{k r}{2}\right)^{2 m+1} g_{m}, \\
g_{m}=\left\{\begin{array}{ll}
(-1)^{m / 2} & : m=\text { even number } \\
(-1)^{(m+1) / 2} & : m=\text { odd number }
\end{array} .\right.
\end{gathered}
$$

Details of the derivation are described in Tasaka et al. (2015). We performed numerical experiments to evaluate the influence of measurement noise using this exact 
Table 1 Evaluation results of viscosity $\mu$ and elasticity $E$, with phase difference in linear viscoelasticity $\delta$ for the differently filtered data of $u(r, t)$ for measurements ranging from $r / R=0.86-0.96$ over a period of $4 \mathrm{~s} ; N_{t}$ and $N_{s}$ correspond to the number of time and space points used in filtering

\begin{tabular}{llllll|llllll}
\hline$\#$ & $N_{t}$ & $N_{s}$ & $\mu[\mathrm{Pa} \cdot \mathrm{s}]$ & $E[\mathrm{~Pa}]$ & $\delta[\mathrm{deg}]$ & $\#$ & $N_{t}$ & $N_{s}$ & $\mu[\mathrm{Pa} \cdot \mathrm{s}]$ & $E[\mathrm{~Pa}]$ & $\delta[\mathrm{deg}]$ \\
\hline 1 & 15 & 19 & 1.05 & 69.6 & 84.57 & 5 & 15 & 11 & 0.70 & 46.2 & 84.54 \\
2 & 15 & 17 & 0.99 & 64.8 & 84.55 & 6 & 15 & 9 & 0.58 & 37.9 & 84.54 \\
3 & 15 & 15 & 0.91 & 60.3 & 84.56 & 7 & 35 & 15 & 0.02 & 1.2 & 84.49 \\
4 & 15 & 13 & 0.82 & 53.6 & 84.54 & 8 & 27 & 15 & 0.39 & 125.9 & 84.52 \\
\hline
\end{tabular}

solution, Eq. 10 with as sufficiently large number of terms and $f_{0}=\omega /(2 \pi)=1 \mathrm{~Hz}$.

To represent measurement noise, and in particular the typical spikey noise seen in UVP measurements, the Mersenne twister method (Saito and Matsumoto (2008)) was used to artificially generate the random noise by modifying the standard deviation $\sigma_{\mathrm{N}}$ in regard to noise level. The cost function to be minimized is derived from Eq. 9 in differential form,

$$
\begin{aligned}
F(\nu)=\sum_{r, t}\left[\frac{u_{i+1, j}-u_{i, j}}{\Delta t}\right. & -\nu\left(\frac{u_{i, j+1}-2 u_{i, j}+u_{i, j-1}}{\Delta r^{2}}\right. \\
& \left.\left.+\frac{u_{i, j+1}-u_{i, j}}{2 r_{j} \Delta r}-\frac{u_{i, j}}{r_{j}^{2}}\right)\right]^{2} .
\end{aligned}
$$

The correct value for the kinematic viscosity is set at $\nu_{0}=1000 \mathrm{~mm}^{2} / \mathrm{s}$, and the cost function is calculated over the range $\nu=800-1200 \mathrm{~mm}^{2} / \mathrm{s}$ in increments of $\Delta \nu=4 \mathrm{~mm}^{2} / \mathrm{s}$ over radial range $r / R=0.95-0.98$. The dependence of the cost function on $\nu$ (Fig. 2) is calculated from the exact solution without noise using Eq. 11; note that $F(\nu)$ has been normalized by the number of velocity data, $N_{\text {total }}$, used for the calculation. In the range explored for $\nu$, the cost function has a unique minimum corresponding to $\nu_{0}$ signifying that the methodology to evaluate $\nu$ from spatio-temporal velocity distributions works well in instances without noise.

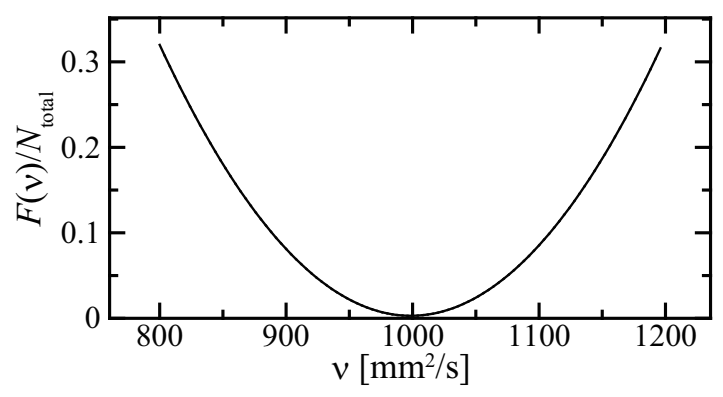

Fig. 2 Variation of the cost function with $\nu$ around minimum setting value of $\nu_{0}=1000 \mathrm{~mm}^{2} / \mathrm{s}$
In assessing the influence of noise on the evaluation of $\nu$, the variation of the cost function is investigated near where it takes a minimum value. Here Gaussian noise of zero-mean is generated at every data point of $u(r, t)$, and is added to the velocity data. Noise level $\sigma_{\mathrm{N}}$ is given as the fraction normalized by the local maximum velocity $U_{\max }(r)$. Actual noise level on $u(r, t)$ measured by UVP is at least larger than $0.1 \%$ $\left(\sigma_{\mathrm{N}} / U_{\max }(r)>10^{-3}\right)$. The results obtained from the velocity data calculated from the exact solution in Eq. 10 with different time resolutions, $\Delta t, f_{0} \Delta t=0.03$ and 0.005, are plotted in Fig. 3. Here fitted parabolic curves, obtained using the least-squares method, have been superimposed on the plots. The viscosities evaluated decrease monotonically with respect to $-\sigma_{\mathrm{N}}^{2}$ for both time resolutions, and have large deviations even at relatively small noise levels of around $0.01 \%$ in $\sigma_{\mathrm{N}} / U_{\max }(r)$. For larger $\Delta t$, there is less influence from the added noise because enhancements of noise contributed by the numerical differentials is smaller.

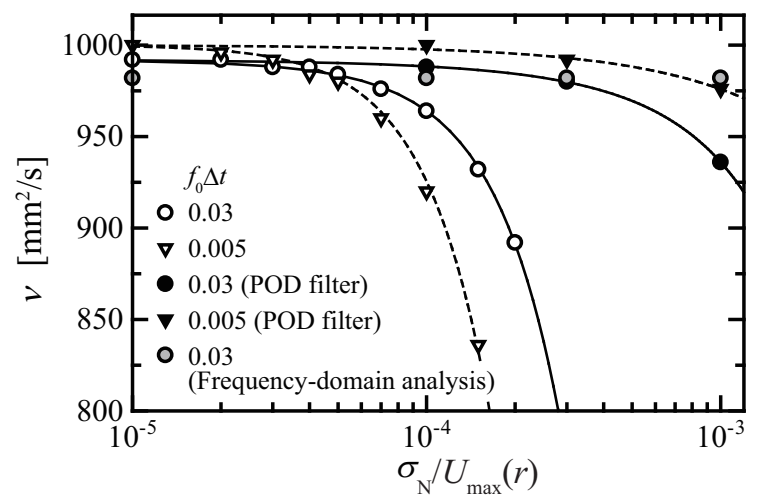

Fig. 3 Estimated values of $\nu$ for different noise levels $\sigma_{\mathrm{N}}$ obtained using two different sampling times, w/o POD filtering and frequency domain analysis; the solid and dashed lines represent fitted parabolic curves of the plots obtained using the least-squares approximation

The monotonic decrease of $\nu$ may be explained as larger noise amplification in the radial derivative in Eq. 9, especially 2nd-order derivative (the other two 
terms produce no strong impact on noise transfer). The radial derivative term in Eq. 11 always has a larger deviation from the correct values without noise than the time derivative term, because of the 2nd-order derivative in the radial term. To satisfy the balance of equation, the estimated $\nu$ must be smaller. By simplifying the equation for the cost function, Eq. 11, this is modeled simply as

$F(\Delta \nu, \epsilon)=\sum\left[A-\left(\nu_{0}+\Delta \nu\right) B \epsilon\right]^{2}$

where $A$ and $B$ are values of the differential calculations in condition without noise, so that $A \approx \nu_{0} B$, and $\Delta \nu$ and $\epsilon$ are the deviations from $\nu$ and the noise amplification rate, $\epsilon>1$. The local minimum of the cost function is found to be $\nu_{0}+\Delta \nu$ satisfying the relation, $\frac{\partial F}{\partial \Delta \nu}=-2 \sum A B \epsilon+2\left(\nu_{0}+\Delta \nu\right) \sum B^{2} \epsilon^{2}=0$.

Assuming $A=\nu_{0} B$, the estimated value from the condition above is a fraction of the correct value of $\nu, \nu_{0}$, specifically,

$\frac{\nu_{0}+\Delta \nu}{\nu_{0}}=\frac{\sum B^{2} \epsilon}{\sum B^{2} \epsilon^{2}}$.

This fraction is always smaller than unity. That is, the monotonic decreasing trend in $\nu$ stems from the enhancement in noise from the 2nd-order derivative in the radial direction.

From Fig. 3, the influence of measurement noise on the evaluation of $\nu$ is significant and non-negligible, and therefore eliminating noise from the velocity data is required. From the standard treatment of measured UVP data, filterng based on the (snapshot) proper orthogonal decomposition (POD)(Sirovich (1987)) provides an effective solution in achieving this purpose (Takeda (1999); Furuichi et al. (2003); Tasaka et al. (2016)). POD is good and objective at extracting organized flow structures. In the present configuration, the flows are well-organized structures and unorganized components are filtered out as noise. From Fig. 3, the present POD filtering can suppress the influence of noise, but there is still a certain influence of noise remaining in the evaluation. Here we remark that, after the filtering, data with fine time resolution provides much better results than rougher ones. This is because the enhancement of noise by taking numerical differentials is reduced by POD filtering, and the fine time resolution now can provide better estimation of the differentials.

\section{Frequency domain analysis}

As examined above, the influence of noise and its enhancement using numerical differentials cannot be avoided completely by filtering the velocity data. Instead, here we propose a novel algorithm to evaluate rheological properties from the velocity data employing the equation of motion as method of analysis in the frequency domain free of difference calculation.

\subsection{Theory}

Taking the Fourier transform with respect to $t$, Eq. 3 becomes

$\hat{\tau}+i \omega \frac{\mu}{E} \hat{\tau}=\mu\left(\frac{\partial \hat{u}}{\partial r}-\frac{\hat{u}}{r}\right)$,

where, the Fourier transform is denoted

$\hat{\tau}(r, \omega)=\mathcal{F}[\tau(r, t)], \hat{u}(r, \omega)=\mathcal{F}[u(r, t)]$.

The Fourier transform changes the differential equation into an algebraic equation that can be solved for $\hat{\tau}$,

$\hat{\tau}(r, \omega)=\frac{\mu\left(\frac{\partial \hat{u}}{\partial r}-\frac{\hat{u}}{r}\right)\left(1-i \omega \frac{\mu}{E}\right)}{1+\left(\omega \frac{\mu}{E}\right)^{2}}$.

Cauchy's equation of motion, Eq. 1, is also converted into

$i \omega \rho \hat{u}=\left(\frac{\partial}{\partial r}+\frac{2}{r}\right) \hat{\tau}$.

Using Eqs. 16 and 17, finding $\mu$ and $E$ becomes an optimization problem for the cost function,

$F(E, \mu ; r)=\int_{0}^{\Omega}\left[i \omega \rho \hat{u}-\left(\frac{\partial}{\partial r}+\frac{2}{r}\right) \hat{\tau}\right]^{2} d \omega$.

That is, $\mu$ and $E$ are determined by $\min _{\mu, E} F(E, \mu ; r)$. Inside the square bracket of Eq. 18 is a complex function that needs to be decomposed into its real and imaginary parts for the numerical calculation. We define,

$\hat{\tau}(r, \omega)=\frac{\mu}{1+\left(\omega \frac{\mu}{E}\right)^{2}}\left[R_{e}(r, \omega)+i I_{m}(r, \omega)\right]$,

where

$R_{e}(r, \omega)=\partial_{r} \Re[\hat{u}]-\frac{1}{r} \Re[\hat{u}]+\omega \frac{\mu}{E}\left(\partial_{r} \Im[\hat{u}]-\frac{1}{r} \Im[\hat{u}]\right)$,

$I_{m}(r, \omega)=\partial_{r} \Im[\hat{u}]-\frac{1}{r} \Im[\hat{u}]-\omega \frac{\mu}{E}\left(\partial_{r} \Re[\hat{u}]-\frac{1}{r} \Re[\hat{u}]\right)$.

Substituting these into Eq. 18, the integrand becomes

$$
\begin{aligned}
{\left[i \omega \rho \hat{u}-\left(\frac{\partial}{\partial r}+\frac{2}{r}\right) \hat{\tau}\right]^{2} } & =\left[\omega \rho \Im[\hat{u}]+\Gamma\left(\frac{\partial}{\partial r}+\frac{2}{r}\right) R_{e}\right]^{2} \\
& +\left[\omega \rho \Re[\hat{u}]-\Gamma\left(\frac{\partial}{\partial r}+\frac{2}{r}\right) I_{m}\right]^{2},
\end{aligned}
$$

where

$\Gamma=\frac{\mu}{1+\left(\omega \frac{\mu}{E}\right)^{2}}$. 


\subsection{Viscometry}

To evaluate the applicability of the frequency-domain analysis proposed in this study, we perform a viscosity analysis on both velocity data created from the exact solution in Eq. 10 with artificial noise and UVP measurement data.

Taking the Fourier transform of the equation for Newtonian fluids yields

$i \omega \hat{u}=\nu D_{r} \hat{u}, \quad \hat{u}=R_{u}(r, \omega)+i I_{u}(r, \omega)$.

The cost function defined in Eq. 18 becomes

$$
\begin{aligned}
F(\nu ; r) & =\int_{\Omega}\left[i \omega \hat{u}-\nu D_{r} \hat{u}\right]^{2} d \omega \\
& =\int_{\Omega}\left[\left(\nu D_{r} R_{u}+\omega I_{u}\right)^{2}+\left(\omega R_{u}-\nu D_{r} I_{u}\right)^{2}\right] d \omega .
\end{aligned}
$$

The actual flows in the USR system are time periodic and discrete Fourier transform (DFT) is applicable for the analysis. From the definition of the Fourier series expansion,

$u(r, t)=\frac{a_{0}(r)}{2}+\sum_{k=1}^{N}\left[a_{k}(r) \cos \Delta \omega k t+b_{k}(r) \sin \Delta \omega k t\right]$

where

$\Delta \omega=2 \pi \Delta f$

the cost function is expressed as

$F(\nu ; r)=\sum_{k=1}^{N}\left(A_{k}^{2}+B_{k}^{2}\right)$

where,

$A_{k}(r)=\Delta \omega k b_{k}-\nu D_{r} a_{k}, B_{k}=\Delta \omega k a_{k}+\nu D_{r} b_{k}$.

The range of frequencies (or range of $k$ ) for the summation above is determined from the sampling frequency on $u(r, t), \Delta f$, and the number of data, $N$ from $\Delta f$ $(k=1)$ to $N \Delta f(k=N)$. Taking a wide range of frequencies, however, would induce noise caused by measurement error that is not related to the cylinder oscillation. A narrow range, $k_{1} \Delta f<f_{0}<k_{2} \Delta f$, is set around the driving frequency of the cylinder oscillation $f_{0}$. The cost function is little modified as

$F_{f_{0}}(\nu ; r)=\sum_{k=k_{1}}^{k_{2}}\left(A_{k}^{2}+B_{k}^{2}\right)$.
To avoid the propagation of measurement noise caused by the numerical differentials of $r$ included in the differential operator $D_{r}$, an $M$ th power series approximation on $a_{k}(r)$ and $b_{k}(r)$ is introduced,

$a_{k}(r)=\sum_{m=0}^{M} \alpha_{m} r^{m}, \quad b_{k}(r)=\sum_{m=0}^{M} \beta_{m} r^{m}$.

Substituting this into definitions of $A_{k}$ and $B_{k}$, Eq. 26, provides

$A_{k}(r)=\Delta \omega k \sum_{m=0}^{M} \beta_{m} r^{m}-\nu \sum_{m=0}^{M}\left(m^{2}-1\right) \alpha_{m} r^{m-2}$
$B_{k}(r)=\Delta \omega k \sum_{m=0}^{M} \alpha_{m} r^{m}+\nu \sum_{m=0}^{M}\left(m^{2}-1\right) \beta_{m} r^{m-2}$.

We perform numerical experiment as in $\S 3.2$ to check the applicability of the present theory and procedure. The velocity data is processed by DFT, and $a_{k}$ and $b_{k}$ in the Fourier series are approximated by fifth-order polynomials (i.e., $M=5$ in Eq. 28). In the power spectrum of $\sqrt{a_{k}^{2}+b_{k}^{2}}$, there is sharp peak corresponding to the oscillation frequency $f_{0}$. Because the frequency resolution, $\Delta f$, is determined by the number of data and the time resolution, the peak frequency $f_{\mathrm{c}}$ does not always coincide with $f_{0}$ and spreads over several frequencies. In the present case, $f_{\mathrm{c}}=1.0101 \mathrm{~Hz}$ and three frequencies nearby contribute more than $97 \%$ of the total fluctuation in kinetic energy. Within the frequency band, $k_{1}-k_{2}$ in Eq. 27, two conditions, $f=f_{\mathrm{c}} \sim$ $f_{0}$ and $f=f_{\mathrm{c}} \pm \Delta f$, are examined. Nevertheless, there is no quantitative difference in values of $\nu$ associated with the conditions. Hence we adopt the condition $f=f_{\mathrm{c}}$, from here on. The results of evaluation of kinematic viscosity at different noise levels are summarized in Fig. 3 with the original results (without noise treatment) and the POD-filtered velocity data. The value of $\nu$ evaluated in the present frequency-domain analysis stays the same value regardless of the noise level, whereas the others decrease with increasing noise level. The frequencydomain analysis provides slightly smaller values than the others and the correct value, $\nu_{0}=1000 \mathrm{~mm}^{2} / \mathrm{s}$, in relatively low noise conditions. This is mainly caused by small disagreement between $f_{\mathrm{c}}$ and $f_{0}$ due to the sampling frequency and sampling data number of $u(r, t)$. Nevertheless, it produces the small deviation even at relatively large noise levels than expected in actual situations with UVP measurements. 


\subsection{Application of viscometry}

The frequency-domain analysis is applied on spatiotemporal velocity measurement data obtained from silicone oil $\left(\nu=300 \mathrm{~mm}^{2} / \mathrm{s}\right.$ at $\left.25^{\circ} \mathrm{C}\right)$ and presented in Yoshida et al. (2017) with an evaluation of its kinematic viscosity using phase information. A 145-mm-diameter acrylic cylinder filled with oil was periodically oscillated with $f_{0}=1 \mathrm{~Hz}$ frequency and through an $80^{\circ}$ angular amplitude. The measurement line of the velocity profiles was set at $\Delta y=15 \mathrm{~mm}$; the UVP was performed with a $30-\mathrm{ms}$ time resolution and a $0.62-\mathrm{mm}$ spatial resolution (measurement direction). Fig. 4 shows the spatio-temporal velocity distribution over three oscillation cycles. The velocity variation appears smooth compared with typical velocity profile measurements, considerable roughness is apparent in comparison with ideal velocity profiles.

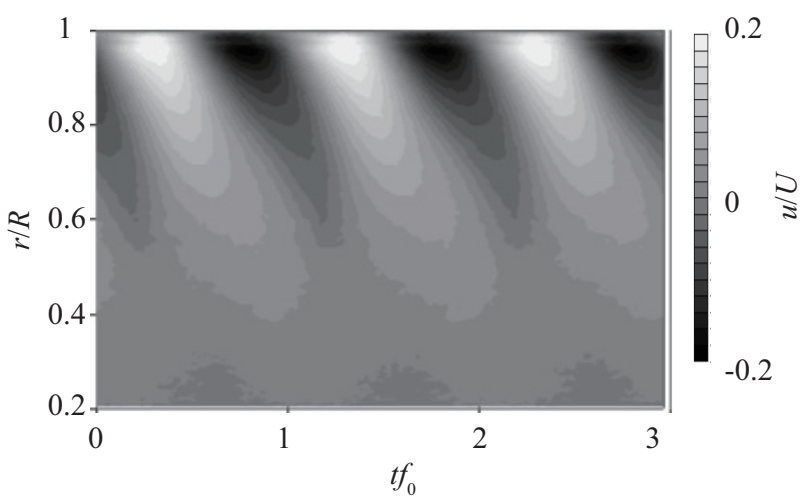

Fig. 4 Spatio-temporal velocity distribution measured for $300 \mathrm{~mm}^{2} / \mathrm{s}$ silicone oil in a cylinder oscillating at $f_{0}=1 \mathrm{~Hz}$ frequency through an $80^{\circ}$ angular amplitude

We processed the velocity distribution using the DFT method, and obtained Fourier coefficients $a_{k}$ and $b_{k}$ as radial profiles. Then a fifth-order polynomial fitting was performed on the profiles with $f=f_{\mathrm{c}} \approx f_{0}$ to ensure a smooth radial dependence. An unavoidable characteristic of the UVP measurement is relatively large deviations near the boundaries around $r / R=1$. The deviations appear mainly in $b_{k}(r)$, and therefore we have omitted these points in the fitting. The fitted curves approximate the plotted data well apart from data points for $b_{k}$ near the cylinder wall. Following the process established in the last section, the cost function, Eq. 27, was calculated at each radial position in the range $\nu$, $100 \leq \nu \leq 500$ and is displayed as a distribution over the $\nu-r$ plane (Fig. 6). The local viscosity is taken to be the local minimum of the cost function at each radial position. The radial profiles of the cost function form a "valley" with $\nu$ uniquely determined. This valley is shallower in the inner region of the cylinder because the amplitude of the velocity fluctuations is smaller and information is not sufficient for the evaluation.

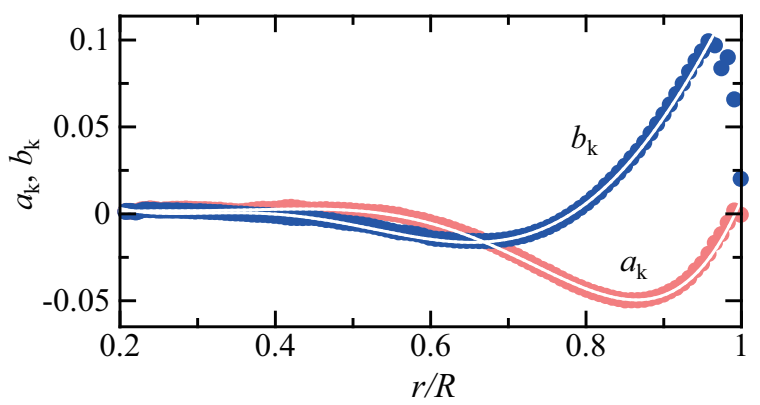

Fig. 5 Fifth-order polynomial fittings (white curve) of the radial profiles for $a_{k}$ and $b_{k}$, where red and blue plots represent the original discrete values of $a_{k}$ and $b_{k}$, respectively

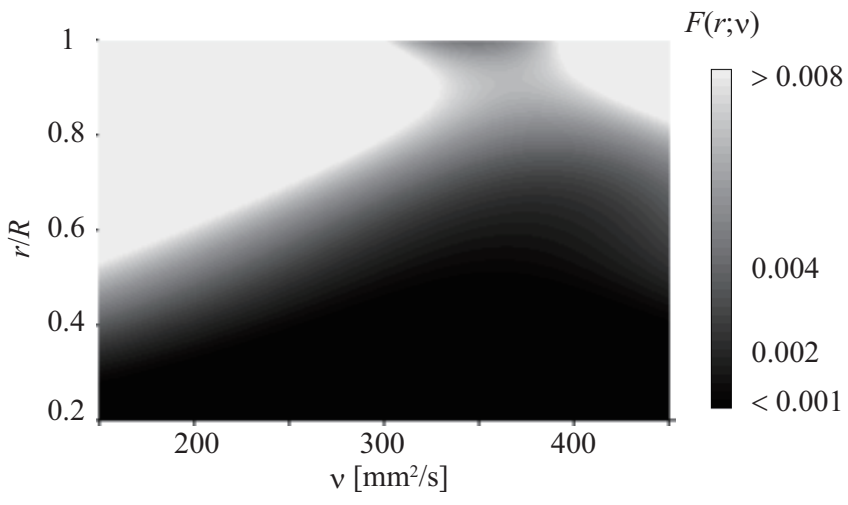

Fig. 6 Gray-scale distribution of the cost function $F(r ; \nu)$

From the frequency-domain analysis, the radial profile of $\nu$ was estimated (Fig. 7) using the polynomial approximation and compared with $\nu$ estimated from a phase-slope analysis on the same data given in Yoshida et al. (2017). The local slope of the phase delay of the velocity fluctuation with respect to the cylinder oscillation reflects the local viscosity, and comparing the phase slopes obtained from the analytical solution, Eq. 10, and experimental data yields the local kinematic viscosity. Similar values were obtained although with deviations; $\nu$ given by the phase analysis exhibit large deviations in the interior of the cylinder, whereas that obtained from the frequency-domain analysis yields similar values. The phase analysis also employs the Fourier transform and suppresses the influence of measurement error on the velocity fluctuations. Nevertheless, the phase information extracted from very small velocity fluctuations is not representative of the flow, and hence calculations using numerical differentials propagate errors. In contrast, profiles of the Fourier coefficients at the main 
frequency retain almost all information in representing the flow and thus this method provides an advantage when analyzing viscometric data. Further, returning to the original purpose of the study, the frequency-domain analysis is applicable to linear viscoelastic analysis using rheological model. We remark that the evaluation of the kinematic viscosity from the experimental data of $300 \mathrm{~mm}^{2} / \mathrm{s}$ oil using the cost function in Eq. 11 is unable to determine the local minimum of $\nu$ over the range, $100 \leq \nu \leq 500$ even with POD filtering.

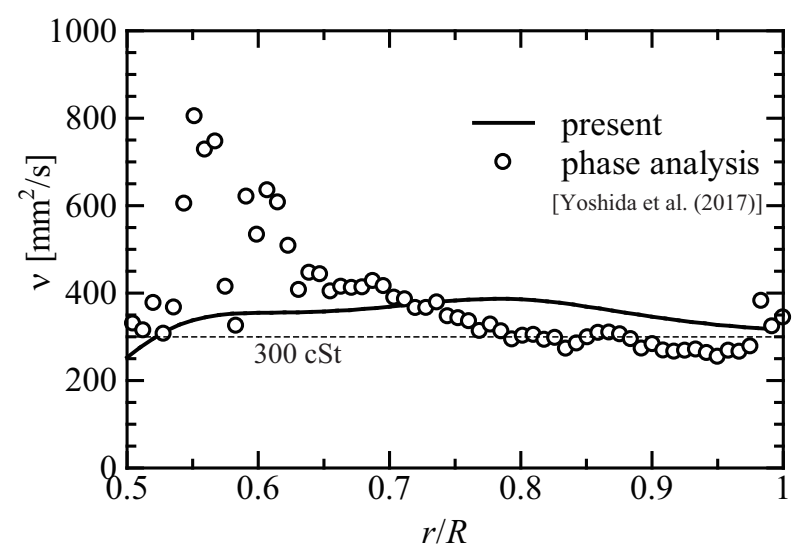

Fig. 7 Radial profiles of the estimated kinematic viscosity from a frequency-domain analysis (solid line) and for comparison a phase profile analysis (circles) from Yoshida et al. (2017)

\subsection{Linear viscoelastic analysis of bubble suspension}

According to the same idea on the viscometry adopting Fourier series expansion of $u(r, t)$ (Eq. 23) and power series approximation on the Fourier coefficients (Eq. 28), the cost function for the linear viscoelastic analysis in Eq. 18 is modified into

$F_{f_{0}}(E, \mu ; r)=\sum_{k=k_{1}}^{k_{2}}\left(A_{k}^{2}+B_{k}^{2}\right)$

where

$$
\begin{aligned}
A_{k}(r) & =\sum_{m=0}^{M}\left[-\rho \Delta \omega k \beta_{m} r^{m}\right. \\
& \left.+\Gamma\left(m^{2}-1\right)\left(\alpha_{m}-\Delta \omega k \mu \beta_{m} / E\right) r^{m-2}\right], \\
B_{k}(r) & =\sum_{m=0}^{M}\left[\rho \Delta \omega k \alpha_{m} r^{m}\right. \\
& \left.+\Gamma\left(m^{2}-1\right)\left(\beta_{m}+\Delta \omega k \mu \alpha_{m} / E\right) r^{m-2}\right] .
\end{aligned}
$$

The algorithm is examined on an analysis of a polyacrylamide aqueous solution (1 wt \%), which has shearrate-dependent viscosity and elasticity, to check its applicability for more complex fluids before performing linear viscoelastic analysis of bubble suspensions. Spatiotemporal velocity information was captured in the same system of oscillating cylinder (see Fig. 1) that was also used in our previous studies (Tasaka et al. (2015); Yoshida et al. (2017)). The setting parameters for the oscillation are $f_{0}=1 \mathrm{~Hz}$ in the frequency and $\Theta=90^{\circ}$ in the amplitude. For the analysis, the velocity data was processed by DFT method to derive Fourier coefficients $a_{k}(r)$ and $b_{k}(r)$ in Eqs. 32 and 33 corresponding to the Fourier component of $f=f_{0}$. Fifth-order power series approximation is then adopted to approximate their radial profiles. In evaluation of the cost function in Eq. 31 for the linear viscoelastic analysis, the phase difference in linear viscoelastic analysis $\delta$ is used as a parameter instead of $E$. Viscosity $\mu$ and $\delta$ are obtained at each radial position by the analysis as shown in Fig. 8; the values are plotted against the amplitude of shear-rate variations $\dot{\gamma}_{0}$, which is given from the Fourier coefficients and calculated using power series approximation in Eq. 28 as

$\dot{\gamma}_{0}=\sqrt{\left[\sum_{m=0}^{M}(m-1) \alpha_{m} r^{m-1}\right]^{2}+\left[\sum_{m=0}^{M}(m-1) \beta_{m} r^{m-1}\right]^{2}}$.

In the figure, the viscosity calculated by phase profile analysis (Yoshida et al. (2017)) from the same velocity data is also plotted for comparison. The viscosity evaluated by the linear viscoelastic analysis gradually decreases with $\dot{\gamma}_{0}$, and expresses shear thinning characteristics of the solution. Along the decrease of viscosity, $\delta$ approaches to $90^{\circ}$ meaning that the solution loses elastic property toward pure viscous body.

We now come back to the linear viscoelastic analysis of a bubble suspension by the frequency domain analysis established above. Fourier coefficient $a_{k}(r)$ and $b_{k}(r)$ in Eqs. 32 and 33 are given by DFT method performed on the velocity data of the bubble suspension used in $\S 3.1$. Then fifth-order power series approximation is performed on radial profiles of the coefficients corresponding to $f=f_{\mathrm{c}} \approx f_{0}(1 \mathrm{~Hz})$. The profiles are approximated well as shown in Fig. 9. In the calculation of the cost function, phase delay of the linear viscoelasticity described in Eq. 8 is changed as a parameter instead of $E$ in range from $50^{\circ}$ to $90^{\circ}$ with increment of $0.1^{\circ}$. Exploring range of $\mu$ was set around the original viscosity of base liquid of the suspension, $1000 \mathrm{~mm}^{2} / \mathrm{s}$ silicone oil, $\mu_{0}=0.97 \mathrm{~Pa} \cdot \mathrm{s}$, from 0.5 to $1.5 \mathrm{~Pa} \cdot \mathrm{s}$ with increment of $0.005 \mathrm{~Pa} \cdot \mathrm{s}$. 


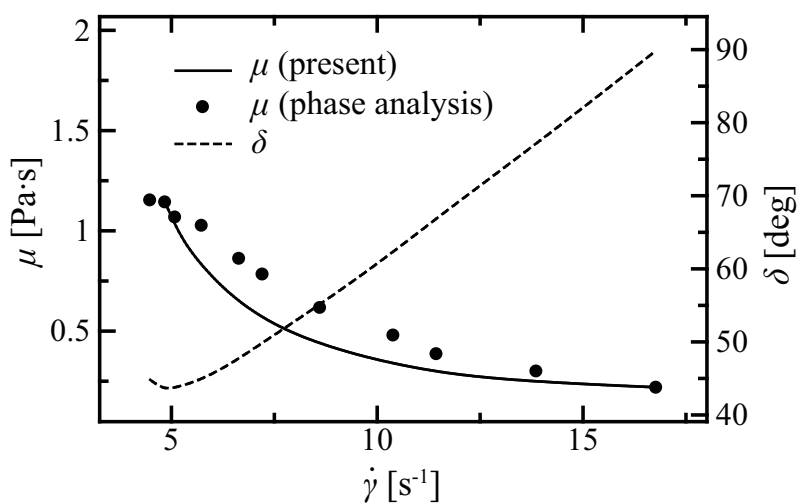

Fig. 8 Variations of viscosity $\mu$ and phase difference in linear viscoelasticity $\delta$ with respect to shear rate $\dot{\gamma}$ for $1 \mathrm{wt} \%$ polyacrylamide aqueous solution, where $\mu$ calculated by the phase profile analysis (Yoshida et al. (2017)) from the same velocity data is also plotted

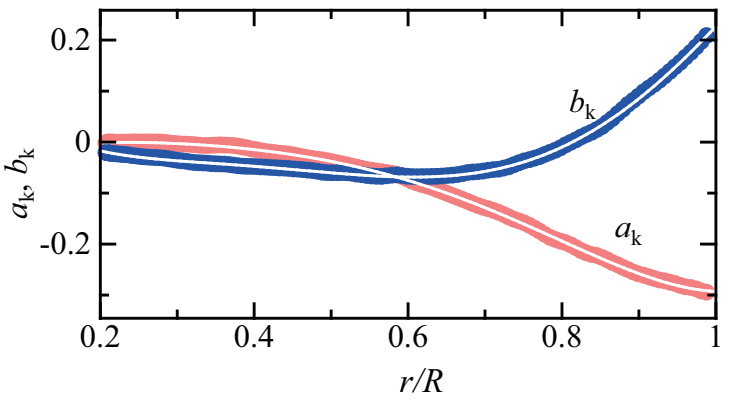

Fig. 9 Fifth-order polynomial fittings (white curve) of the radial profiles for $a_{k}$ and $b_{k}$ obtained in the bubble suspension, where red and blue plots represent the original discrete values of $a_{k}$ and $b_{k}$, respectively

An example of the calculated cost function is shown in Fig. 10 as distribution of $F$ on $\delta-\mu$ plane for $r=$ $0.9 R$. The distribution is expressed in logarithmic gray scale, and there is single local minimum point; values of $\delta$ and $\mu$ are given as values on this point. At least in the range of $\delta$ and $\mu$ we examined, the cost function increases monotonically from the local minimum point, and the values are uniquely given. For $r / R<0.9$ the local minimum point attaches to the boundary of $\delta=$ $90^{\circ}$, but uniqueness of the solution is unchanged.

Results of estimations of $\delta$ and $\mu$ according to the cost function calculated at each radial position from $r / R=0.6$ to 0.95 are summarized in Table 2. Elasticity $E$ is calculated through Eq. 8 with $\mu$ and $f_{0}$ in cases that $\delta \neq 90^{\circ} .\left|\mu^{*}\right|$ denotes effective complex viscosity defined as

$\left|\mu^{*}\right|=\frac{\mu}{\sqrt{1+(\omega \mu / E)^{2}}}$.

The linear viscoelastic analysis separated influences of unsteady bubble deformations in the oscillating shear flows on the momentum propagation into viscous and

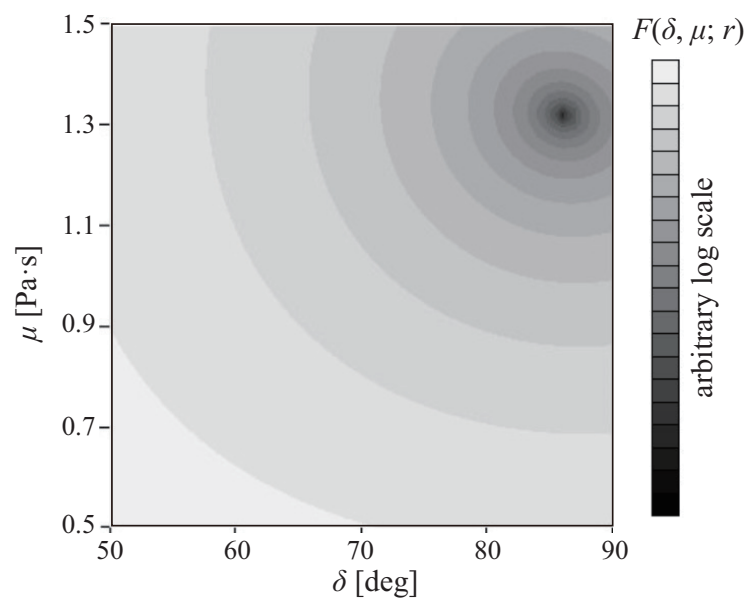

Fig. 10 Gray-scale distribution of the cost function, $F(\delta, \mu ; r=0.9 R)$ for the bubble suspension

elastic contributions. In the table, $\delta$ takes $90^{\circ}$ without a region $r / R \geq 0.9$, and the elastic contribution appears in the region. This is reasonable because capillary number exceeds effectively the critical capillary number to allow bubble deformations in this range, and the deformation bubbles existing nearby the wall show considerable deformations (Tasaka et al. (2015)). In the oscillating shear flows, the bubbles experience periodically strong shear and relaxation. The elastic effect may be provided by restoring the original spherical shape in the relaxation. Effective viscosity normalized by original viscosity of the oil, $\left|\mu^{*}\right| / \mu_{0}$, distributes around unity within the deviation order of volume fraction of bubbles, $2 \%$ for $r / R \leq 0.8$. This is in good agreement with knowledge of effective viscosity for spherical bubbles.

Table 2 Evaluation results of viscosity $\mu$, phase difference in linear viscoelasticity $\delta$, and elasticity $E$ calculated from $\mu$ and $\delta$ through Eq. 8 at different radial position $r / R$, where the amplitude of local shear-rate variation $\dot{\gamma}_{0}$ is calculated from the velocity data through Eq. 34, and $\left|\mu^{*}\right|$ indicates effective viscosity normalized by the original viscosity value of the oil, $\mu_{0}=0.97 \mathrm{~Pa} \cdot \mathrm{s}$

\begin{tabular}{llllll}
\hline$r / R$ & $\dot{\gamma}_{0}\left[\mathrm{~s}^{-1}\right]$ & $\mu[\mathrm{Pa} \cdot \mathrm{s}]$ & $\delta[\mathrm{deg}]$ & $E[\mathrm{~Pa}]$ & $\left|\mu^{*}\right| / \mu_{0}$ \\
\hline 0.95 & 15.89 & 1.46 & 77.1 & 39.8 & 1.46 \\
0.9 & 14.28 & 1.32 & 86.1 & 121.2 & 1.36 \\
0.85 & 12.46 & 1.17 & 90.0 & - & 1.21 \\
0.8 & 10.53 & 1.05 & 90.0 & - & 1.08 \\
0.75 & 8.63 & 0.99 & 90.0 & - & 1.02 \\
0.7 & 6.90 & 0.97 & 90.0 & - & 0.99 \\
0.65 & 5.45 & 0.99 & 90.0 & - & 1.02 \\
0.6 & 4.32 & 1.05 & 90.0 & - & 1.08 \\
\hline
\end{tabular}




\section{Conclusion}

To achieve a stable evaluation of the linear viscoelasticity in bubble suspensions as complex multiphase fluids using USR, we assessed the influence of measurement noise enhancement in the evaluation of rheological properties using the equation of motion with measurement data of spatio-temporal velocity fluctuations. By avoiding calculations of numerical differential using velocity data, a frequency-domain analysis was proposed for applications in linear viscoelastic analysis. By taking the Fourier transform of the equation of motion and the constitutive equations (the rheological models), time derivatives are converted to algebraic calculations, and further, approximating the radial profiles of Fourier coefficients by finite power series enables an evaluation of the rheological properties without requiring calculation of numerical differentials. In rheology, this novel combination of techniques was scrutinized using numerical experiments by considering noise artificially generated in viscometric data. The present method provided better estimates of viscosity in comparison with dealing with raw velocity data and POD filtering. The technique was also applied to actual velocity data measured in $300 \mathrm{~mm}^{2} / \mathrm{s}$ silicone oil as a Newtonian fluid, and the estimations were reasonable agreement with previous results. The frequency-domain analysis was then extended to the linear viscoelastic analysis and its applicability was examined on polyacrylamide aqueous solution, which is a shear thinning, viscoelastic fluid. The analysis finally achieved separation of influences of unsteady bubble deformation into viscous and elastic contributions on the momentum propagation.

The algorithm proposed here is aptly applicable in a wider range of velocity-profiling rheometry. And, it may be able to support to evaluate, e.g., fluids taking shear banding effects and solutions with distributions of concentration.

\section{Acknowledgment}

This work was supported by JSPS KAKENHI Grant No. $17 \mathrm{H} 01245$.

\section{References}

Ancey C (2005) Solving the Couette inverse problem using a wavelet-vaguelette decomposition. J Rheol 49:441-460.

Ceccio SL (2010) Friction drag reduction of external flows with bubble and gas injection. Ann Rev Fluid Mech 42:183-203.

Choi SJ, Schowalter WR (1975) Rheological properties of nondilute suspensions of deformable particles. Phys Fluids 18:420-427.
Derakhshandeh B, Vlassopoulos D (2012) Thixotropy, yielding and ultrasonic Doppler velocimetry in pulp fiber suspensions. Rheol Acta 51:201-214.

Dimitriou CJ, Casanellas L, Ober TJ, Mckinley GH (2012) Rheo-PIV of a shear-banding wormlike micellar solution under large amplitude oscillatory shear. Rheol Acta 51:395-411.

Doi M, Ohta T (1991) Dynamics and rheology of complex interfaces. J Chem Phys 95:1242-1247.

Einstein A (1906) Eine neue Bestimung der Molekuldimensionen. Ann Phys 19:289-306.

Fischer S, Schmitt P, Ensminger D, Abda F, Pallares A (2008) A new velocity estimation method using spectral identification of noise. Flow Meas Instr 19:197-203.

Frankel NA, Acrivos A (1970) The constitutive equation for a dilute emulsion. J Fluid Mech 44:65-78.

Furuichi N, Takeda Y, Kumada M (2003) Spatial structure of the flow through an axisymmetric sudden expansion. Exp Fluids 34:643-650.

Gurung A, Haverkort JW, Drost S, Norder B, Westerweel J, Poelma C (2016) Ultrasound image velocimetry for rheological measurements. Meas Sci Technol 27:094008.

Heirman G, Hendrickx R, Vandewalle L, Gemert DV, Feys D, Schutter GD, Desmet B, Vantomme J (2008) Integration approach of the Couette inverse problem of powder type self-compacting concrete in a wide-gap concentric cylinder rheometer. J Non-Newtonian Fluid Mech 150:93-103.

Jarny S, Roussel N, Rodts S, Bertrand F, Roy RL, Coussot P (2005) Rheological behavior of cement pastes from MRI velocimetry. Cement Concrete Res 35:1873-1881.

Llewellin EW, Manga M (2005) Bubble suspension rheology and implications for conduit flow. J Volcanol Geotherm Res 143:205-217.

Llewellin EW, Mader HM, Wilson SDR (2002) The constitutive equation and flow dynamics of bubbly magmas. Geophys Res Lett 29:2170.

Meacci V, Ricci S, Wiklund J, Birkhofer B, Kotzé R (2016) Flow-Viz - An integrated digital in-line fluid characterization system for industrial applications. in IEEE Sensors Applications Symposium (SAS) 2016:1-6.

Murai Y (2012) Ultrasonic Doppler velocity profiler for fluid flow. Takeda Y, ed. 101 Springer, Tokyo: 153-156.

Murai Y (2014) Frictional drag reduction by bubble injection. Exp Fluids 55:1773.

Murai Y, Oiwa H (2008) Increase of effective viscosity in bubbly liquids from transient bubble deformation. Fluid Dyn Res 40:565-575.

Muramatsu E, Murakawa H, Sugimoto K, Asano H, Takenaka N, Furuichi N (2015) Multi-wave ultrasonic Doppler method for measuring high flow-rates using staggered pulse intervals. Meas Sci Technol 27:025303.

Ouriev B, Windhab EJ (2002) Rheological study of concentrated suspensions in pressure-driven shear flow using novel in-line ultrasound Doppler method. Exp Fluids 32: $204-211$.

Ovarlez G, Bertrand F, Rodts S (2005) Local determination of the constitutive law of a dense suspension of noncolloidal particles through magnetic resonance imaging. J Rheol 50:259-292.

Pérez-González J, López-Durán JJ, Marín-Santibánez BM, Rodríguez-González F (2012) Rheo-PIV of a yield-stress fluid in a capillary with slip at the wall. Rheol Acta 51:937-946.

Quinzani LM, Armstrong RC, Brown RA (1995) Use of coupled birefringence and LDV studies of flow through a planar contraction to test constitutive equations for concen- 
trated polymer solutions. J Rheol 39:1201-1228.

Rao MA, Rizvi SSH, Datta AK, Ahmed J eds (2014) Engineering Properties of Foods, Fourth Edition (CRC press, Boca Raton): 661-663.

Rodríguez-González F, Pérez-González J, de Vargas L, Marín-Santibánez BM (2010) Rheo-PIV analysis of the slip flow of a metallocene linear low-density polyethylene melt. Rheol Acta 49:145-154.

Rothstein JP, Mckinley GH (2002) Inhomogeneous transient uniaxial extensional rheometry. J Rheol 46:1419-1443.

Rust AC, Manga M (2002) Effects of bubble deformation on the viscosity dilute suspensions. J Non Newton Fluid Mech 104:53-63.

Saito M, Matsumoto M (2008) SIMD-oriented Fast Mersenne Twister: a 128-bit Pseudorandom Number Generator. in Monte Carlo and Quasi-Monte Carlo Methods 2006, ed. by L'Ecuyer P, Owen AB Springer, Berlin Heidelberg: 607-622.

Salmela J, Haavisto S, Koponen A, Jäsberg A, Kataja M (2013) Rheological characterization of micro-fibrillated cellulose fibre suspension using multi scale velocity profile measurements. in Proceeding of 15th Fundamental Research Symposium.

Savizky A, Golay MJE (1964) Smoothing and differentiation of data by simplified least square procedures. Analytical Chemistry 36:1627-1639.

Serrano-Aguilera JJ, L. Parras L, del Pino C, RubioHernandez FJ (2016) Rheo-PIV of Aerosil@) R816/polypropylene glycol suspensions. J NonNewtonian Fluid Mech 232: 22-32.

Shiratori T, Tasaka Y, Murai Y (2016) Rapid rheological characterization of viscoelastic fluid based on spatiotemporal flow velocimetry. Exp Therm Fluid Sci 71: 1-13.

Shiratori T, Tasaka Y, Murai Y, Takeda Y (2013) Development of ultrasonic visualizer for capturing the characteristics of viscoelastic fluids. J Vis 16:275-286.

Shiratori T, Tasaka Y, Oishi Y, Murai Y (2015) Ultrasonic velocity profiling rheometry based on a widened circular Couette flow. Meas Sci Technol 26:085302.

Sirovich L (1987) Turbulence and the dynamics of coherent structures. part I: Coherent structures. Quart Applied Math 45:561-571.

Stickel JJ, Powell RL (2005) Fluid mechanics and rheology of dense suspensions. Annu Rev Fluid Mech 37:129-149.

Takeda Y (1999) Quasi-periodic state and transition to turbulence in a rotating Couette system. J Fluid Mech 389:8199.

Takeda Y, ed. (2012) Ultrasonic Doppler velocity profiler for fluid flow. Springer, Tokyo.

Tasaka Y, Kimura T, Murai Y (2015) Estimating the effective viscosity of bubble suspensions in oscillatory shear flows by means of ultrasonic spinning rheometry. Exp Fluids 56: $1-13$

Tasaka Y, Igaki K, Yanagisawa T, Vogt T, Zuerner T, Eckert S (2016) Regular flow reversals in Rayleigh-Bénard convection in a horizontal magnetic field. Phys Rev E 93: 043109 .

Taylor GI (1932) The viscosity of a fluid containing small drops of another fluid. Proc R Soc Lond Ser A 138:41-48.

Wiklund J, Stading M (2008) Application of in-line ultrasound Doppler-based UVP-PD rheometry method to concentrated model and industrial suspensions. Flow Meas Instrum 19:171-179.

Yeow YL, Ko WC, Tang PP (2000) Solving the inverse problem of Couette viscometry by Tikhonov regulation. J Rheol 44:1335-1351.
Yoshida T, Tasaka Y, Murai Y (2017) Rheological evaluation of complex fluids using ultrasonic spinning rheometry in an open container. J Rheol 61:537-549. 